\title{
c-Abl kinase regulates neutrophil extracellular trap formation and lung injury in abdominal sepsis
}

\author{
Avin Hawez ${ }^{1}$, Zhiyi Ding ${ }^{1}$, Dler Taha ${ }^{1}$, Raed Madhi $\mathbb{D}^{1}{ }^{1}$, Milladur Rahman $\mathbb{D}^{1}$ and Henrik Thorlacius ${ }^{1 凶}$ \\ (c) The Author(s), under exclusive licence to United States and Canadian Academy of Pathology 2021
}

Sepsis is associated with exaggerated neutrophil responses although mechanisms remain elusive. The aim of this study was to investigate the role of c-Abelson (c-Abl) kinase in neutrophil extracellular trap (NET) formation and inflammation in septic lung injury. Abdominal sepsis was induced by cecal ligation and puncture (CLP). NETs were detected by electron microscopy in the lung and by confocal microscopy in vitro. Plasma levels of DNA-histone complexes, interleukin-6 (IL-6) and CXC chemokines were quantified. CLP-induced enhanced phosphorylation of c-Abl kinase in circulating neutrophils. Administration of the c-Abl kinase inhibitor GZD824 not only abolished activation of c-Abl kinase in neutrophils but also reduced NET formation in the lung and plasma levels of DNA-histone complexes in CLP mice. Moreover, inhibition of c-Abl kinase decreased CLP-induced lung edema and injury. Administration of GDZ824 reduced CLP-induced increases in the number of alveolar neutrophils. Inhibition of c-Abl kinase also markedly attenuated levels of CXC chemokines in the lung and plasma as well as IL- 6 levels in the plasma of septic animals. Taken together, this study demonstrates that c-Abl kinase is a potent regulator of NET formation and we conclude that c-Abl kinase might be a useful target to ameliorate lung damage in abdominal sepsis.

Laboratory Investigation (2022) 102:263-271; https://doi.org/10.1038/s41374-021-00683-6

\section{INTRODUCTION}

Abdominal sepsis is a life-threatening condition caused by an exaggerated and misdirected host responses characterized by wide-spread activation of innate immune cells ${ }^{1}$. Neutrophils are recognized to play a key role in sepsis ${ }^{2,3}$. On one hand, neutrophils are critical for eliminating invading microorganisms but on the other hand, excessive neutrophil responses cause organ damage and failure ${ }^{4}$. Convincing data have shown that neutrophil recruitment constitute a rate-limiting step in septic lung injury ${ }^{2,5}$. Beside secretion of antimicrobial compounds and phagocytic killing $^{6}$, neutrophils can eliminate pathogens through expulsion of neutrophil extracellular traps (NETs) composed of neutrophilderived DNA forming extracellular web-like structures decorated with nuclear histones as well as granular and cytoplasmic proteins ${ }^{7,8}$. NETs and their associated histones have been demonstrated to cause epithelial and endothelial cell damage ${ }^{9}$. Moreover, NETs provoke several pro-inflammatory effects in the blood $^{10}$, pancreas ${ }^{11}$, liver ${ }^{12}$, and lung ${ }^{10}$. In fact, defective clearance of NETs increases organ damage in sepsis ${ }^{13}$. Although NETs seem to be important in the pathophysiology, the signaling mechanisms controlling NET generation in sepsis are not known.

Activation of neutrophils is mediated by multiple signaling pathways converging on specific transcription factors regulating adhesion and migration ${ }^{2,14}$. Many of these signaling cascades are controlled by intracellular kinases phosphorylating down-stream targets ${ }^{15}$. c-Abelson (c-Abl) kinase is a ubiquitously expressed nonreceptor tyrosine-protein kinase, which was initially identified as a potent driver of myeloid cell transformation into leukemia ${ }^{16}$. More recent studies have shown that c-Abl kinase also plays a role in inflammation. For example, inhibition of c-Abl kinase protects against endotoxemic lung injury ${ }^{17,18}$, vascular leakage ${ }^{19,20}$, and IgG-induced glomerular injury ${ }^{21}$. In addition, it has been reported that c-Abl kinase regulates $\beta_{2}$-integrin-mediated neutrophil migration via activation of $\operatorname{Vav} 1^{22,23}$. Knowing that reactive oxygen species (ROS) are involved in the expulsion of NETs, it is interesting to note that c-Abl kinase has been implicated in the neutrophil formation of $\operatorname{ROS}^{24}$. Thus, accumulating data indicate that c-Abl kinase plays important functions in neutrophils and in several models of inflammation, which warrant further studies on the role of c-Abl kinase in NET formation and septic lung injury.

Based on these considerations, we hypothesized that c-Abl kinase activity might be involved in NET formation and lung damage in abdominal sepsis.

\begin{abstract}
METHODS Animals

All experiments were conducted in accordance with the legislation on the protection of animals and approved by the Regional Ethical Committee for Animal Experimentation at Lund University, Sweden (Permit number: 5.8.18-08769/2019). Male C57BL/6 mice (20-25 g) were kept in a pathogen free facility on a 12-12 $\mathrm{h}$ light-dark cycle with free access to food and tap water. Mice were housed at least one week before use at a maximum of five mice per cage with environment enrichment. Animals were anesthetized with $75 \mathrm{mg}$ of ketamine hydrochloride (Hoffman-La Roche, Basel, Switzerland) and $25 \mathrm{mg}$ of xylazine (Janssen Pharmaceutica, Beerse, Belgium) per kg body weight. The ARRIVE guidelines were consulted for all animal experiments ${ }^{25}$.
\end{abstract}

${ }^{1}$ Department of Clinical Sciences, Malmö, Section for Surgery, Skåne University Hospital, Lund University, 20502 Malmö, Sweden. ${ }^{\circledR}$ email: henrik.thorlacius@med.lu.se 


\section{Experimental protocol of sepsis}

Abdominal sepsis model was induced by cecal ligation and puncture (CLP) as previously described ${ }^{26}$. Briefly, animals were anesthetized and the abdominal wall was incised in the midline to mobilize the cecum. The cecum was filled with feces from ascending colon, ligated $75 \%$ of cecum (5-0 silk suture), soaked with PBS and punctured twice with a 21-gauge needle. The cecum was then returned to the peritoneal cavity and the abdominal incision was sutured. The specific C-ABL kinase inhibitor GZD824 (5 mg/kg, Selleck Chemicals, Houston, TX, USA) or vehicle (DMSO) was administered via the jugular vein $30 \mathrm{~min}$ prior to induction of abdominal sepsis. Sham mice underwent the identical laparotomy and resuscitation procedures, but the cecum was neither ligated nor punctured. Mice were then returned to their cages and reanesthetized for sample collection at 6 and $24 \mathrm{~h}$ after CLP induction. The left lung was ligated and excised for edema measurement. The right lung was used for collecting bronchoalveolar lavage fluid (BALF), in which neutrophils were quantified in a Burker chamber. Next, the lung was perfused with PBS, one part was fixed in formaldehyde for histology and the remaining lung tissue was snap-frozen in liquid nitrogen, and stored at $-80^{\circ} \mathrm{C}$ for later enzymelinked immunosorbent assay (ELISA) as described subsequently.

\section{Isolation of blood neutrophils}

Blood was collected from the inferior vena cava in acid citrate dextrose (1:10). Blood samples were added to Roswell Park Memorial Institute medium 1640 (RPMI 1640, Invitrogen, Stockholm, Sweden) supplemented with $10 \%$ fetal bovine serum (FBS, Invitrogen) and $2 \mathrm{mM}$ EDTA (Sigma-Aldrich, Stockholm, Sweden). Following hypotonic lysis $(5 \mathrm{ml}$ ice-cold $0.2 \% \mathrm{NaCl}$, added for $45 \mathrm{~s}$ followed by addition of $5 \mathrm{ml} 1.6 \% \mathrm{NaCl}$ ), neutrophils were separated from mononuclear cells by density gradient centrifugation using a Ficoll-Paque gradient (GE Healthcare, Uppsala, Sweden). The neutrophil layer was isolated and washed with RPMI 1640 and cells were resuspended at $4 \times 10^{6}$ cells $/ \mathrm{ml}$. Next, the cells were homogenized and the activity of c-Abl kinase determined in isolated neutrophils as described below.

\section{Western blot}

Isolated neutrophils were homogenized in ice-cold RIPA buffer (RIPA Lysis and Extraction Buffer, ThermoFisher, USA) containing protease inhibitors (Halt Protease Inhibitor Cocktail; Pierce Biotechnology, Rockford, IL) for 20 min and then sonicated and centrifuged $\left(16,000 \mathrm{~g}\right.$ for $\left.15 \mathrm{~min}, 4^{\circ} \mathrm{C}\right)$. Supernatants were collected and stored at $-20^{\circ} \mathrm{C}$. Protein concentration of supernatants were determined by use of Pierce BCA Protein Assay Reagent (Pierce Biotechnology). Proteins $(20 \mu \mathrm{g}$ per lane) were separated by $8-16 \%$ Mini-PROTEAN ${ }^{\circ}$ TGX Stain-Free ${ }^{T M}$ Gels (Bio-Rad) and transferred to polyvinylidene fluoride membranes (Novex, San Diego, CA, USA). Before blotting, total protein gel images were taken using Bio-Rad's stain-free gel chemistry. Membranes were blocked in TBS/Tween 20 buffer containing $5 \%$ non-fat dry milk powder. Protein immunoblots were performed using rabbit anti-c-Abl kinase (2862, 1:1000, Cell signaling technology, Danvers, USA) and biotin-conjugated anti-phosphotyrosine antibody $4 \mathrm{G} 10^{\circ}$ Platinum (16-452, 1:1000, Merckmillipore, Darmstadt, Germany) antibodies. Membranes were first incubated with HRP-conjugated anti-biotin secondary antibody (7075P5, 1:1000, Cell Signaling, Leiden, Netherlands) for detection of phosphor-c-Abl kinase. For detection of total c-Abl kinase same membranes were stripped off first and then incubated with antirabbit HRP-conjugated secondary antibody (7074, Cell Signaling, Leiden, Netherlands). Precision Plus Protein ${ }^{T M}$ standards (1610363, BIO-RAD, Hercules, CA, USA) and Precision Protein ${ }^{T M}$ StrepTactin-HRP conjugate (1610380, BIO-RAD) were used to determine the molecular weight of the c-Abl kinase. Protein bands were developed and analyzed using the BioRad ChemiDoc $^{\text {TM MP }}$ imaging system. Image Lab $^{\text {TM }}$ software version 5.2.1. was used to normalize target protein band signal against the total protein of respective lane before ratio calculation (Suppl. Fig. 1).

\section{Histology}

Lung samples were fixed by immersion in $4 \%$ formaldehyde phosphate buffer overnight and then dehydrated and paraffin-embedded. Six $\mu \mathrm{m}$ sections were stained with haematoxylin and eosin. Lung injury was quantified in a blinded manner by adoption of a preexisting scoring system as previously described ${ }^{27,28}$ including size of alveolar spaces, thickness of alveolar septae, alveolar fibrin deposition and neutrophil infiltration graded on a zero (absent) to four (extensive) scale. Five random areas were scored and mean value was used. Histology score was the sum of all four parameters.

\section{Lung edema}

Lung edema formation was measured using the lung wet-to-dry weight ratio. The left lung was excised, washed in PBS, gently dried using a blotting paper and weighed. The tissue was then dried at $60^{\circ} \mathrm{C}$ for $72 \mathrm{~h}$ and re-weighed. The change in the ratio of wet weight to dry weight was used as indicator of lung edema formation.

\section{BALF}

Animals were placed supine and the trachea was exposed by dissection. An angiocatheter was inserted into the trachea. BALF was collected by five washes of $1 \mathrm{ml}$ ice-cold PBS containing $5 \mathrm{mM}$ EDTA. The numbers of monomorphonuclear (MNL) and polymorphonuclear (PMNL) cells were counted in a Burker chamber.

\section{Systemic leukocyte count}

Blood was collected from tail vein and mixed with Turks solution $0.2 \mathrm{mg}$ gentian violet in $1 \mathrm{~mL}$ glacial acetic acid; $6.25 \% \mathrm{vol} / \mathrm{vol}$ ) in a $1: 20$ dilution. Leukocytes were counted as MNL and PMNL leukocytes in a Burker chamber.

\section{ELISA}

Plasma and lung levels of CXCL1, CXCL2 and IL-6 (R\&D Systems, Abingdon, UK, Siemens, Marburg, Germany) were measured $24 \mathrm{~h}$ after induction of CLP. Linearity was assessed and confirmed by serial dilution of standards containing recombinant mouse CXCL1, CXCL2 and IL-6 in a calibrator diluent.

\section{Transmission electron microscope and scanning electron microscope}

Deparaffinized lung tissue samples were fixed in $2.5 \%$ glutaraldehyde in $0.15 \mathrm{~mol} / \mathrm{L}$ sodium cacodylate, $\mathrm{pH} 7.4$ (cacodylate buffer), for $30 \mathrm{~min}$ at room temperature. Specimens were washed with cacodylate buffer and dehydrated with an ascending ethanol series from $50 \%$ (vol/vol) to absolute ethanol $(10 \mathrm{~min} / \mathrm{step})$. The specimens then were subjected to critical-point drying in carbon dioxide, with absolute ethanol as intermediate solvent, mounted on aluminum holders, and finally sputtered with $20 \mathrm{~nm}$ palladium/gold. Specimens were examined in a Jeol/FEI XL 30 FEG scanning electron microscope at the Core Facility for Integrated Microscopy at Panum Institute (University of Copenhagen, Denmark). The location of individual target molecules was analyzed at high resolution by ultrathin sectioning and transmission immunoelectron microscopy. Specimens on coverslips were embedded in Epon 812 and sectioned into 50$\mathrm{nm}$-thick ultrathin sections with a diamond knife in an ultramicrotome. For immunohistochemistry, sections were incubated overnight at $4{ }^{\circ} \mathrm{C}$ with primary antibodies against elastase (ab68672, $10 \mu \mathrm{g} / \mathrm{ml}$, Abcam, Cambridge, UK) and citrullinated histone 3 (ab5103, $10 \mu \mathrm{g} / \mathrm{ml}$, Abcam, Cambridge, UK). Controls without primary antibodies were included. The grids then were incubated with species-specific, gold-conjugated secondary antibodies (Electron Microscopy Sciences, Fort Washington, MD). Goldlabeled annexin $\mathrm{V}$ were also used. Finally, the sections were post-fixed in $2 \%$ glutaraldehyde and post-stained with $2 \%$ uranyl acetate and lead citrate. Specimens were observed in a Jeol/FEl CM100 transmission electron microscope operated at $80-\mathrm{kV}$ accelerating voltage at the Core Facility for Integrated Microscopy at Panum Institute.

\section{DNA-histone complexes}

Plasma levels of DNA-histone complexes were quantified by use of a sandwich ELISA based on monoclonal antibodies directed against histones and DNA according to the manufacturer's instructions (Cell Death detection Elisa plus; Roche Diagnostics).

\section{NET formation in vitro}

Bone marrow neutrophils $\left(2 \times 10^{6}\right.$ cells $\left./ \mathrm{ml}\right)$ were freshly isolated by density gradient centrifugation using a Ficoll-Paque gradient (GE Healthcare). Neutrophils were incubated with $100 \mathrm{ng} / \mathrm{ml}$ tumor necrosis factor- $a$ (TNF-a, $100 \mathrm{ng} / \mathrm{ml}$, Peprotech, London, UK) with and without GZD824 (500 nM) for $1 \mathrm{~h}$ at $37^{\circ} \mathrm{C}$. Samples were centrifuged $(400 \mathrm{~g}, 5 \mathrm{~min})$ and supernatants were collected to measure DNA-histone complex as described above. For detection of NETs by flow cytometry, neutrophils were first fixed with $2 \%$ formaldehyde and then washed two times with PBS containing 2\% FBS. Cells were incubated with primary antibodies: PE-conjugated anti-Ly6G 
(551461, $5 \mu \mathrm{g} / \mathrm{ml}$, clone 1A8, BD Pharmingen), fluorescein isothiocyanate conjugated anti-MPO (ab90812, $5 \mu \mathrm{g} / \mathrm{ml}, \mathrm{Abcam}$ ) and rabbit anti-H3cit (citrulline 2,8,17, ab5103, $5 \mu \mathrm{g} / \mathrm{ml}$, Abcam, Cambridge, MA) antibodies in PBS containing 5\% donkey serum. After washing two times, cells were incubated with rat anti-rabbit APC-conjugated secondary antibody (A21038, $5 \mu \mathrm{g} / \mathrm{ml}$, Thermo Scientific, Rockford, IL). For immunofluorescence imaging of NETs, neutrophils were stimulated by TNF-a $(100 \mathrm{ng} / \mathrm{ml})$ with and without GZD824 (500 nM) and stained on glass coverslips as described above. After immunostaining, coverslips were rinsed and mounted in fluoromount with Hoechst 33342 (Thermo Fisher Scientific). Confocal microscopy was performed using LSM 800 confocal (Carl Zeiss, Jena, Germany) by a $\times 63$ oil immersion objective (numeric aperture $=1.25$ ). The pinhole was $\sim 1$ airy unit and the scanning frame was $1024 \times 1024$ pixels. Images were later processed using ZEN2012 software.

\section{ROS formation in neutrophils}

Bone marrow neutrophils were isolated as described above. Neutrophils were incubated with anti-CD16/CD32 (553142, $5 \mu \mathrm{g} / \mathrm{ml}$, BD Pharmingen) to block $\mathrm{FC}_{Y}$ Ill/IIRs and reduce non-specific labeling and APC-conjugated antiLy6G (560599, $5 \mu \mathrm{g} / \mathrm{ml}$, clone 1A8, BD Pharmingen, San Jose, CA) antibodies. To detect ROS (superoxide and hydrogen peroxide) generation, cells were incubated with $1 \mu \mathrm{M}$ dihydroethidium (D23107, Thermo Fisher Scientific) for $15 \mathrm{~min}$ at $37^{\circ} \mathrm{C}$ and then stimulated with $100 \mathrm{ng} / \mathrm{ml} \mathrm{TNF-a} \mathrm{for}$ $20 \mathrm{~min}$ at $37^{\circ} \mathrm{C}$ with and without GZD824 (500 nM). Flow cytometry analysis was performed on a CytoFLEX flow cytometer (Becton Dickinson, Mountain View, CA, USA), and viable gate was used to exclude dead and fragmented cells.

\section{Statistics}

Data are presented as mean values \pm standard error of the mean. For two group comparisons, Mann-Whitney rank sum test was used. $P<0.05$ was considered statistically significant and $n$ represents the number of animals or experiments.

\section{RESULTS}

\section{c-Abl kinase activity in neutrophils}

To determine systemic activation of c-Abl kinase in sepsis, western blot was used to analyze c-Abl phosphorylation in circulating neutrophils from CLP mice. It was found that CLP enhanced phosphorylation of c-Abl kinase in circulating neutrophils (Fig. 1). In addition, treatment with GZD824 markedly decreased c-Abl kinase activation in neutrophils, indicating that GZD824 is an effective inhibitor of c-Abl kinase activity in sepsis (Fig. 1). Injection of GZD824 alone had no effect on c-Abl kinase phosphorylation (Fig. 1).

\section{c-Abl-kinase regulates NETs formation in sepsis}

By use of scanning electron microscopy, we observed that CLP induced formation of extracellular fibrillar and web-like structures in the lung compatible with NETs (Fig. 2A). In addition, transmission immunoelectron microscopy revealed that the neutrophil-derived granule protein elastase and citrillunated histone 3 co-localized with these extracellular fibrillar and weblike structures (Fig. 2B), which were not observed in the normal lung. It was observed that administration of GZD824 greatly reduced generation of NETs in the septic lung (Fig. 2B). Moreover, plasma levels of DNA-histone complexes were increased by eightfold in CLP animals (Fig. 2C). Treatment with GZD824 decreased CLP-induced increases of DNA-histone complexes in plasma by $83 \%$ (Fig. 2C). Administration of GZD824 alone had no effect on NET formation in mice (Fig. 2A-C). To further study the role of c-Abl kinase in regulating NET formation in neutrophils, we used isolated bone marrow neutrophils in vitro. Stimulation of neutrophils with TNF-a markedly increased neutrophil coexpression of neutrophil-derived granule protein MPO and citrullinated histone 3 (Fig. 3A) as well as DNA-histone complexes (Fig. 3B). Indeed, co-incubation of neutrophils with GZD824 dosedependently decreased TNF-a-induced co-expression of MPO and citrullinated histone 3 (Fig. $3 \mathrm{~A}$ ) levels as well as DNA-histone

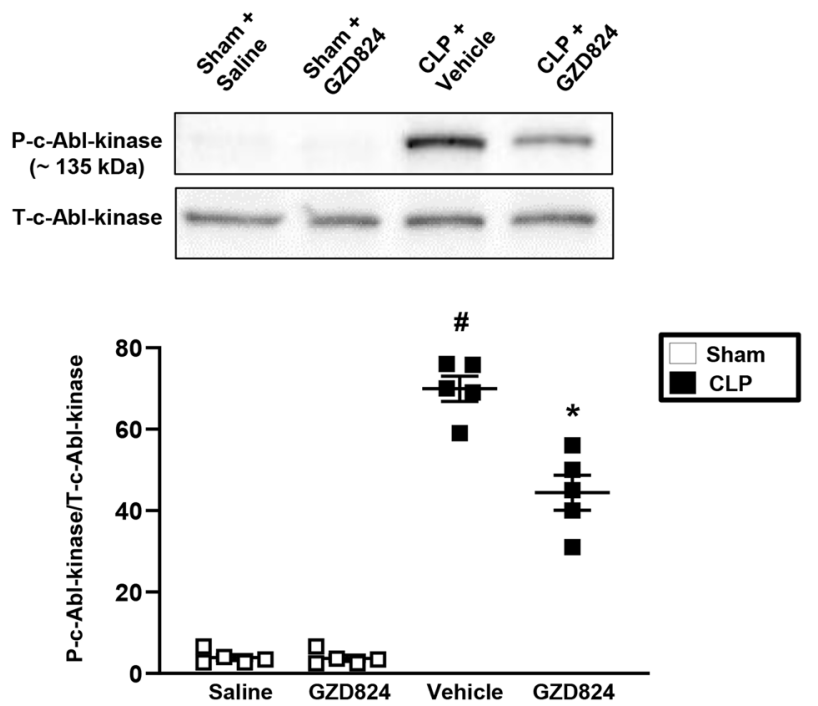

Fig. 1 c-Abl kinase activity in neutrophils. Phosphorylation of c-Abl kinase in circulating neutrophils were examined by western blot as described in Materials and Methods. Animals were treated with GZD824 $(5 \mathrm{mg} / \mathrm{kg})$ or vehicle prior to CLP induction. Mice treated with saline (sham) or GZD824 alone without CLP. Samples were collected $24 \mathrm{~h}$ after induction of CLP. Data are presented as mean values \pm standard error of the mean (SEM) and $n=5$. ${ }^{\#} P<0.05$ vs. Sham and ${ }^{*} P<0.05$ vs. Vehicle + CLP.

complexes (Fig. 3B) in isolated neutrophils, demonstrating that $c-A b l$ kinase regulates NET formation in neutrophils. In addition, using confocal fluorescence microscopy, we found that TNF-a challenge induced formation of DNA fibrillar and web-like structures that co-localized with MPO and citrullinated histone 3 (Fig. 3C). Co-incubation of neutrophils with GZD824 prevented TNF-a-provoked induction of these DNA structures containing MPO and citrullinated histone 3 (Fig. 3 C), suggesting that c-Abl kinase controls NET formation. Knowing that ROS plays a key role in NET formation ${ }^{29}$, it was of interest to study the role of c-Abl kinase in TNF-a-induced formation of ROS. TNF-a stimulation triggered clear-cut formation of ROS in isolated neutrophils (Fig. 4A, B). Co-incubation of neutrophils with GZD824 markedly decreased TNF-a-provoked generation of ROS in neutrophils (Fig. 4A, B).

\section{c-Abl kinase mediates septic lung injury}

Histological analysis showed normal lung architecture in sham mice (Fig. 5A). CLP caused severe pulmonary injury, characterized by destruction of tissue microarchitecture, edema of interstitial tissue, and infiltration of neutrophils (Fig. 5C). Administration of GZD824 decreased CLP-induced tissue destruction and neutrophil infiltration in the lung (Fig. 5D). Quantification revealed that CLPevoked lung damage was reduced in GZD824-treated animals (Fig. 5E). CLP caused clear-cut edema formation in the lung, reflected by an enhanced lung wet-to-dry ratio (Fig. 5F) Treatment with GZD824 decreased lung wet-to-dry ratio by $89 \%$ in septic animals (Fig. 5F).

\section{c-Abl kinase regulates neutrophil recruitment}

The number of BALF neutrophils increased from $21.6 \pm 4.5 \times$ $10^{3}$ in sham mice to $120.0 \pm 24.66 \times 10^{3}$ in CLP mice, corresponding to a 6-fold increase (Fig. 6A). Blocking c-Abl kinase activity decreased the number of BALF neutrophils by $83 \%$ in septic mice (Fig. 6A). Moreover, CLP markedly increased pulmonary formation of CXCL1 and CXCL2 and treatment with GZD824 reduced CXC chemokine generation by more than $89 \%$ and $87 \%$ in CLP animals (Fig. 6B and C). 
A
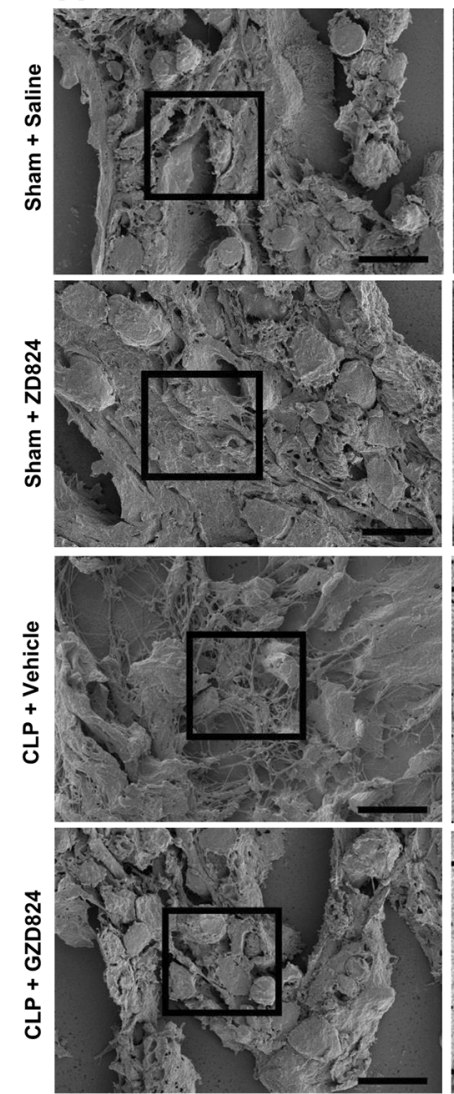

B
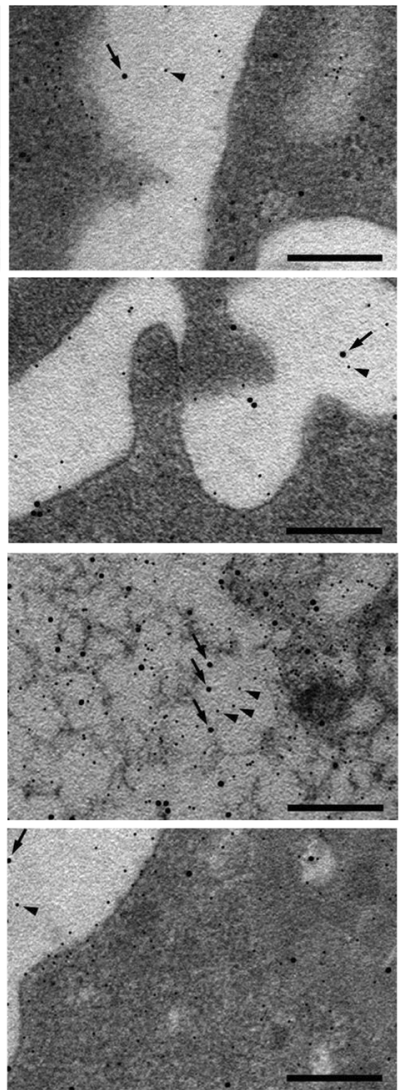

C

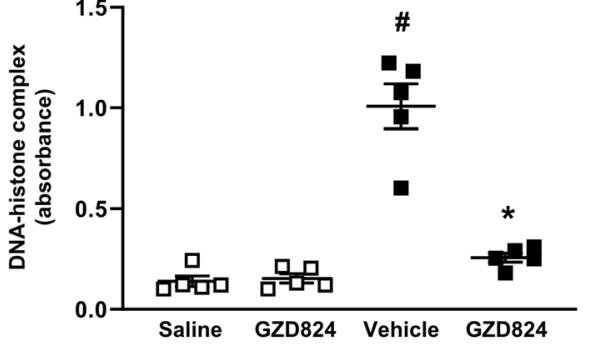

Fig. 2 NET formation in sepsis. A Scanning electron microscopy showing extracellular web-like structures in the lung from CLP mice. Scale bar $=5 \mu \mathrm{m}$. B Transmission electron microscopy of the indicated area of interest from Fig. 2A incubated with gold-labeled antibody against citrullinated histone 3 (large gold particles) and anti-elastase (small gold particles) antibodies. Scale bar $=0.25 \mu \mathrm{m}$. All images are representative of five independent experiments. C DNA-histone complex formation. Animals were treated with GZD824 (5 mg/kg) or vehicle prior to CLP induction. Mice treated with saline (sham) or GZD824 alone without CLP. Samples were collected $24 \mathrm{~h}$ after induction of CLP. Data are presented as mean values \pm standard error of the mean (SEM) and $n=5$. ${ }^{\#} P<0.05$ vs. Sham and ${ }^{*} P<0.05$ vs. Vehicle + CLP.

\section{c-Abl kinase regulates systemic inflammation in sepsis}

Induction of CLP enhanced plasma levels of IL-6 by 29-fold, CXCL1 by 64 -fold and CXCL2 by 37-fold (Fig. 7A-C). Administration of GZD824 decreased CLP-evoked plasma levels of IL-6, CXCL1 and CXCL2 by more than $80 \%, 92 \%$ and $92 \%$, respectively (Fig. $7 \mathrm{~A}-\mathrm{C}$ ). As part of a systemic inflammatory response in sepsis, the number of circulating leukocytes decreases. Indeed, it was observed that CLP caused leukocytopenia (Table 1) and that administration of GZD824 antagonized CLP-induced leukocytopenia in septic mice (Table 1).

\section{DISCUSSION}

Our novel data suggest that c-Abl kinase plays a critical role in the development of abdominal sepsis. These results demonstrate that c-Abl kinase is a key regulator of NET formation in neutrophils and sepsis. In addition, it was found that targeting c-Abl kinase activity not only reduces NET generation but also decreases CLP-induced neutrophil infiltration and lung damage, suggesting that c-Abl kinase might be a useful target in abdominal sepsis.

Convincing data have documented that c-Abl kinase coordinate key aspects of actin dynamics and cytoskeletal rearrangements in cells ${ }^{30,31}$. Most knowledge about the function of c-Abl kinase has been obtained from studies of human leukemias ${ }^{16,32}$ but a growing body of evidence indicate that c-Abl also regulates critical components of inflammation, such endothelial cell integrity and neutrophil adhesion ${ }^{22,23}$. Moreover, the role of
c-Abl kinase has also been implicated in inflammatory diseases, including immunoglobulin-mediated glomerular damage, nephrotoxicity and endotoxin-induced vascular leakage $e^{20,21,33}$. Herein, we found that induction of abdominal sepsis markedly increased c-Abl kinase activity in circulating neutrophils. Administration of GZD824, a specific c-Abl inhibitor, decreased activation of c-Abl kinase in circulating neutrophils in CLP mice, suggesting that GZD824 is an effective inhibitor of c-Abl kinase in vivo. Knowing that neutrophils play a key role in septic lung injury ${ }^{2,34,35}$, we next asked whether c-Abl kinase is involved in CLP-induced pulmonary damage. Indeed, we found that treatment with GZD824 markedly reduced lung edema in abdominal sepsis. These findings are in line with a previous study showing that c-Abl kinase mediates pulmonary vascular leakage and tissue damage triggered by endotoxin $^{18,20}$. Moreover, our data suggest that c-Abl kinase activity regulates induction of septic lung injury, which is in line with a previous report showing that c-Abl kinase regulates sepsisinduced vascular leakage ${ }^{19}$. In this context, it is interesting to note that c-Abl kinase controls downstream activity of small GTPases of the Rho family, such as Rac1 and RhoA ${ }^{36,37}$, which are known to regulate lung injury in abdominal sepsis ${ }^{15,38}$. Thus, it might be speculated that part of the protective effect of inhibiting c-Abl kinase could be related to downstream inhibition of Rac1 or RhoA signaling. Although our data suggest that c-Abl kinase in neutrophils play an important role in septic lung damage, our results do not exclude that c-Abl kinase activity also in other cells are involved in septic lung damage. For example, it has been 
A

B
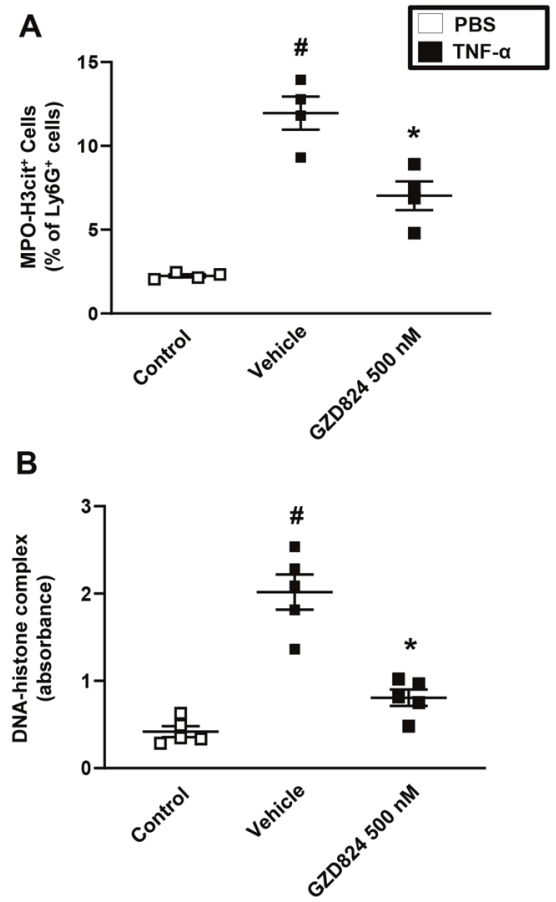

C Hoechst

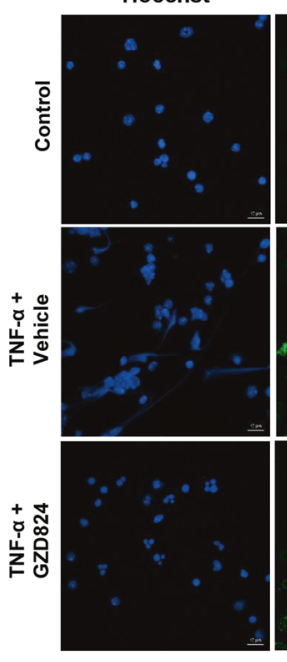

MPO

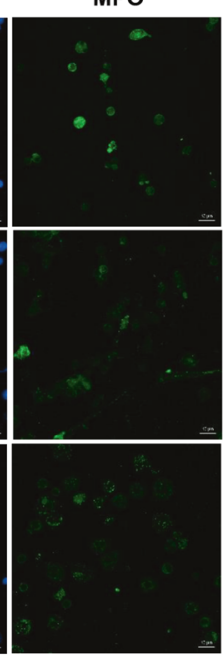

H3cit

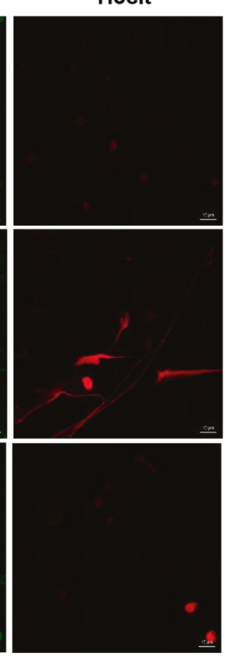

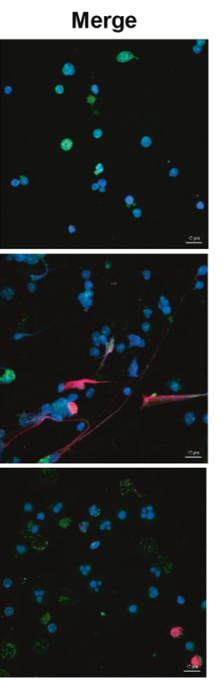

Fig. 3 NET formation in neutrophils. NETs were generated from isolated neutrophils by TNF- $\alpha$-stimulation, co-incubated with or without GZD824 $(500 \mathrm{nM})$. Non-stimulated neutrophils served as a control. A Levels of citrullinated histone 3 and MPO in isolated neutrophils detected by flow cytometry and B DNA-histone complexes in the supernatant determined by ELISA. Data represent means \pm SEM and $n=5$. ${ }^{\#} P<0.05$ versus control mice and ${ }^{*} P<0.05$ versus Vehicle + TNF- $\alpha$. C Neutrophils were immune-stained with antibodies to citrullinated histone 3 (H3cit), myeloperoxidase (MPO), and DAPI nuclear stain. Representative confocal fluorescence microscopy images from four independent experiments. Scale bar indicates $10 \mu \mathrm{m}$.

A

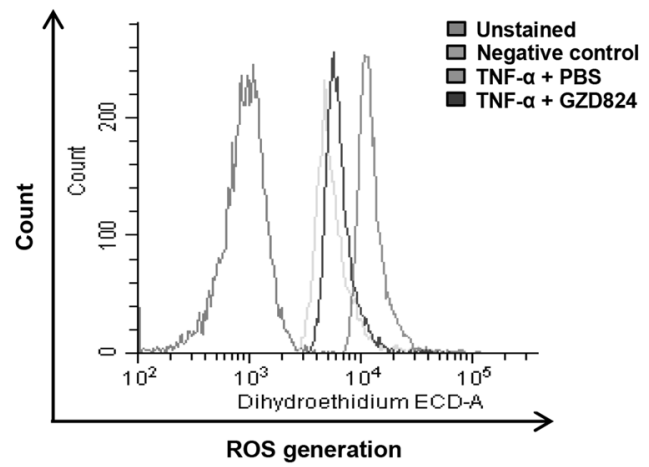

B

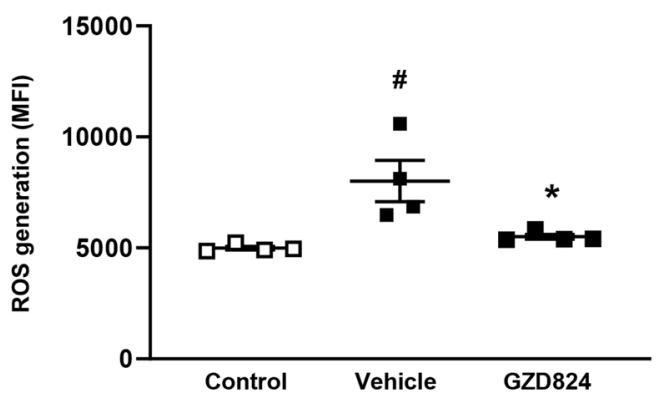

Fig. 4 ROS formation in neutrophils. Quantification of ROS formation in isolated neutrophils by flow cytometry. Neutrophils were stimulated by TNF- $\alpha$ with or without GZD824 $(500 \mathrm{nM})$. Nonstimulated neutrophils served as a control. A Representative histogram of ROS generation and $\mathbf{B}$ aggregate data. Data are presented as mean values \pm standard error of the mean (SEM) and $n=5$. ${ }^{\#} P<0.05$ versus control mice and ${ }^{*} P<$ 0.05 versus Vehicle + TNF- $\alpha$.

shown that c-Abl kinase in endothelial cells plays a key function in regulating vascular permeability induced by pro-inflammatory mediators ${ }^{39}$. In addition, it should be noted that a previous study showed that inhibition of c-Abl kinase attenuates endotoxinprovoked pulmonary inflammation but exacerbates ventilatorinduced lung damage, suggesting that pro-inflammatory effects of c-Abl kinase could be context-dependent ${ }^{40}$.

It is generally held that neutrophil infiltration is a rate-limiting step in septic lung damage ${ }^{2,5}$. For example, depletion of neutrophils or blocking neutrophil recruitment have repeatable been shown to protect against lung damage in abdominal sepsis ${ }^{34,41}$. Herein, it was observed that CLP increased the number of neutrophils in the bronchoalveolar space. Treatment with
GZD824 markedly attenuated number of alveolar neutrophils in the lung, suggesting that c-Abl kinase is a potent regulator of neutrophil recruitment in septic lung injury. Considering the important function of neutrophils in abdominal sepsis ${ }^{2,3,5}$, it could be forwarded that the inhibitory impact of GZD824 on neutrophil accumulation help to explain the protective effect of GZD824 in septic lung damage. Secretion of CXC chemokines, such as CXCL1 and CXCL2, co-ordinates neutrophil trafficking to sites of inflammation ${ }^{42-45}$. CLP triggered massive formation of CXCL1 and $\mathrm{CXCL2}$ in the lung. Administration of GZD824 markedly reduced pulmonary formation of $\mathrm{CXCL1}$ and $\mathrm{CXCL2}$, suggesting that $c-A b l$ kinase regulates CXC chemokine generation in the inflamed lung, which could help to explain part of the inhibitory 
A

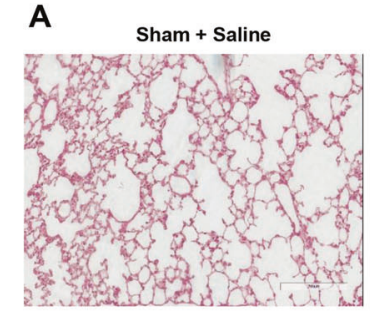

B

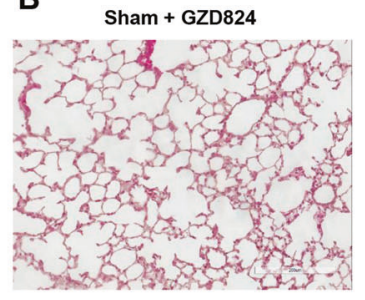

C

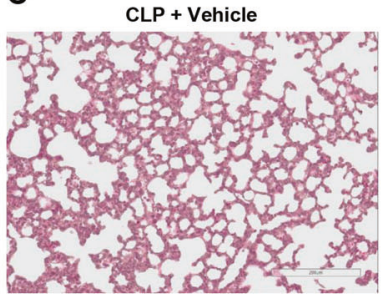

D

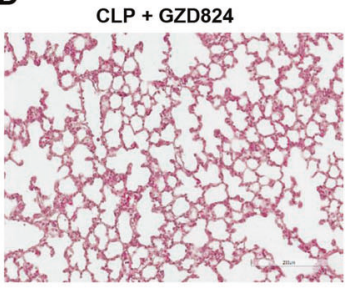

E

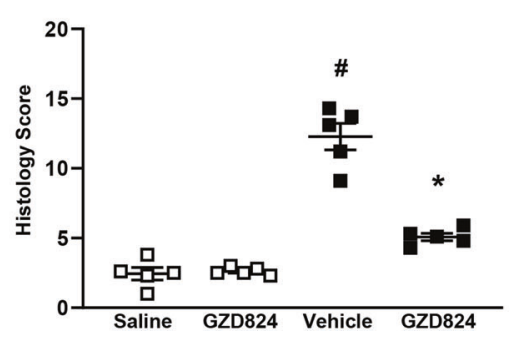

$\mathbf{F}$

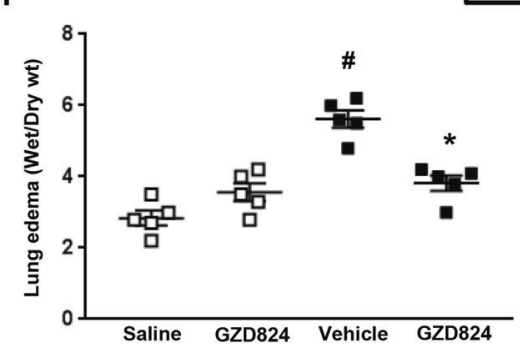

Fig. 5 Representative haematoxylin \& eosin sections of the lung. A Animals were treated with saline or B GZD824 alone. Separate mice were pretreated with C vehicle or D $5 \mathrm{mg} / \mathrm{kg}$ of GZD824 prior to CLP induction. E Lung injury score and $\mathbf{F}$ edema formation were quantified as described in Materials and Methods. Data are presented as mean values \pm standard error of the mean (SEM) and $n=5$. ${ }^{\#} P<0.05$ vs. Sham and ${ }^{*} P<0.05$ vs. Vehicle + CLP. Samples were harvested $24 \mathrm{~h}$ after CLP induction. Scale bar indicates $100 \mu \mathrm{m}$.
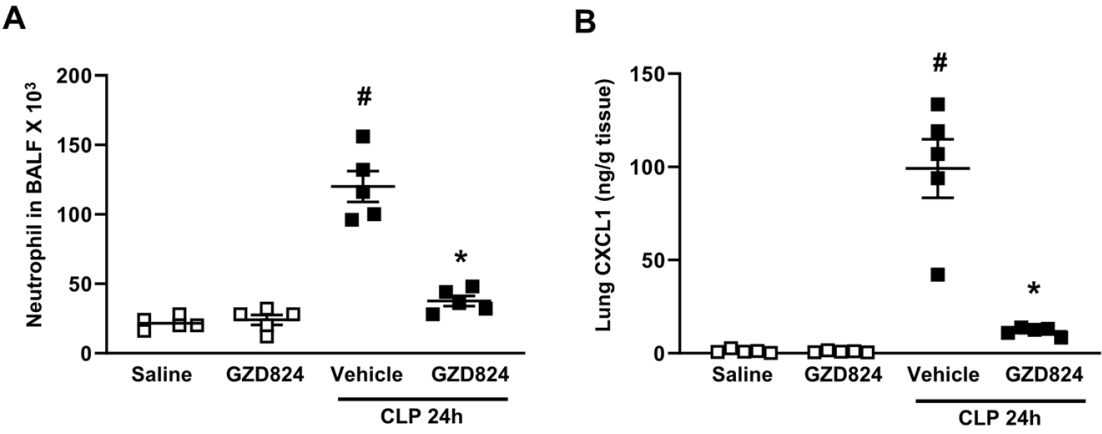

C

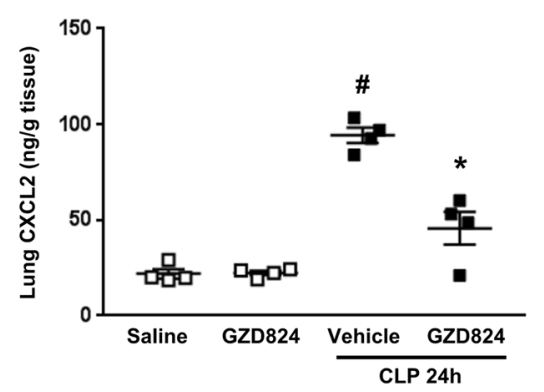

Fig. 6 c-Abl kinase regulates CLP-induced infiltration of neutrophils in the lung. A Number of BALF neutrophils were determined $24 \mathrm{~h}$ after CLP induction. Pulmonary levels of B CXCL1 and C CXCL2. Animals were treated with GZD824 (5 mg/kg) or vehicle prior to CLP induction. Mice treated with saline (sham) or GZD824 alone without CLP. Mice treated with PBS served as sham animals. Data are presented as mean values \pm standard error of the mean (SEM) and $n=5 .{ }^{*} P<0.05$ vs. Sham and ${ }^{*} P<0.05$ vs. Vehicle $+C L P$. 
A

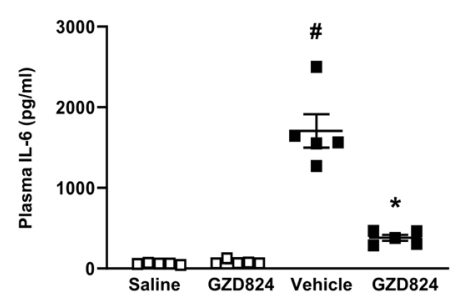

B

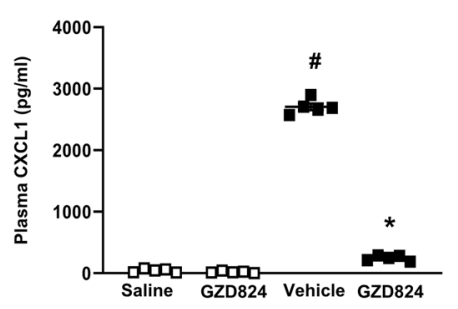

C

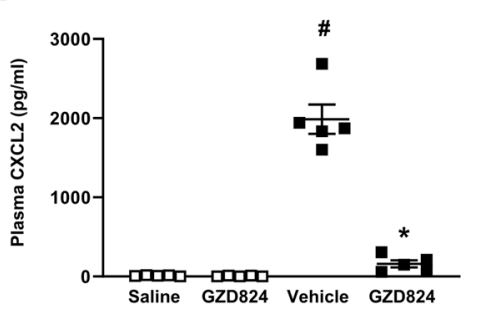

Fig. 7 c-Abl kinase regulates CLP-induced systemic inflammation. Plasma levels of A IL-6, B CXCL1, and C CXCL2. Animals were treated with GZD824 $(5 \mathrm{mg} / \mathrm{kg})$ or vehicle prior to CLP induction. Mice treated with PBS (sham) or GZD824 alone without CLP. Data are presented as mean values \pm standard error of the mean (SEM) and $n=5$. ${ }^{*} P<0.05$ vs. Sham and ${ }^{*} P<0.05$ vs. Vehicle + CLP.

Table 1. Systemic leukocyte differential counts.

\begin{tabular}{|lcll|} 
& MNL & PMNL & Total \\
\hline Sham + Saline & $6 \pm 0.2$ & $1.5 \pm 0.2$ & $7.3 \pm 0.6$ \\
\hline Sham + GZD824 & $5.8 \pm 0.4$ & $1.3 \pm 0.1$ & $7.3 \pm 0.5$ \\
\hline CLP + Vehicle & $1.1 \pm 0.1^{*}$ & $0.4 \pm 0.1^{*}$ & $2.8 \pm 0.2^{*}$ \\
\hline CLP + GZD824 & $3.5 \pm 0.4^{\dagger}$ & $0.9 \pm 0.1^{\dagger}$ & $4.6 \pm 0.7^{\dagger}$
\end{tabular}

Blood was collected from sham animals receiving saline only as well as mice treated with GZD824 $(5 \mathrm{mg} / \mathrm{kg})$ or vehicle intravenously prior to cecal ligation and puncture for $24 \mathrm{~h}$. Cells were identified as monomorphonuclear leukocytes (MNL) and polymorphonuclear leukocytes (PMNL). Data are presented as mean values \pm standard error of the mean (SEM), $10^{6}$ cells/ $\mathrm{ml}$ and $n=5$. ${ }^{*} P<0.05$ vs. Sham and ${ }^{\dagger} P<0.05$ vs. Vehicle + CLP.

impact of GZD824 on neutrophil recruitment in septic lung injury. Leukocytopenia is a hallmark of systemic inflammation ${ }^{35}$. In the present study, it was found that GZD824 antagonized CLPinduced leukocytopenia, indicating that c-Abl kinase also regulates systemic inflammation is sepsis. This notion is also supported by our results showing that GZD824 decreased plasma levels of IL-6, CXCL1, and CXCL2 in septic animals.

Published data in the literature suggest that NETs exert dual roles in infectious diseases. On one hand neutrophil-derived NETs protects against infections by trapping microbes and facilitate interactions between antimicrobial proteins and bacteria leading to microbiological clearance ${ }^{12,46}$. On the other hand, excessive NET generation causes tissue damage in both infectious and noninfectious diseases ${ }^{10,47,48}$. Numerous studies have documented that formation of neutrophil-derived NETs constitute a key component in the pathophysiology of sepsis ${ }^{10,12,49}$. In the present study, we observed that treatment with GZD824 reduced DNA structures co-localizing with the neutrophil-derived granule protein elastase and citrullinated histone 3 in the lung, indicating that c-Abl regulates NET formation in septic lung injury. Moreover, GZD824 decreased CLP-induced formation DNA-histone complexes in the plasma, supporting the notion above that c-Abl plays an important role in NET generation in abdominal sepsis. We then asked whether c-Abl kinase directly regulates NET formation in neutrophils. For this purpose, neutrophils were isolated and challenge with TNF- $a$, a cell wall component of Saccharomyces cerevisiae known to activate c-Abl kinase pathway, which significantly increased expression of MPO and citrullinated histone 3 on expelled neutrophil-derived DNA. Moreover, TNF- $a$ stimulation of isolated neutrophils also enhanced formation DNA-histone complexes. We found that co-incubation with GZD824 decreased TNF-a-induced co-expression of MPO and citrullinated histone 3 on neutrophil-derived DNA as well as DNA-histone complexes in isolated neutrophils, indicating that c-Abl kinase directly controls NET formation in neutrophils. Together, these findings constitute the first evidence in the literature suggesting that c-Abl kinase regulates NET formation in neutrophils and sepsis. Notably, one study has reported that induction of ROS is pivotal in TNF-ainduced NET formation in neutrophils ${ }^{50}$. Herein, we found that inhibition of c-Abl kinase markedly reduced TNF-a-triggered formation of ROS in neutrophils. Thus, c-Abl kinase-dependent generation of ROS might be involved in TNF-a-induced formation of NETs in neutrophils. Although neutrophil-derived NETs are recognized for their important role in the innate immune system by trapping and killing microbes facilitating microbiological clearance $^{12,46}$, several studies have shown that excessive formation of NETs is known to cause tissue injury and organ failure in infectious conditions ${ }^{10,47}$. Thus, pharmacological targeting of c-Abl kinase function might be a useful strategy to treat infectious diseases in which excessive NET formation harm host organs tissue while simultaneously antagonizing microbial pathogens. In this context, it should be noted that timing of anti-inflammatory intervention is critical in infectious diseases considering the important role of the immune system in bacterial clearance unless effective antibiotic coverage is provided throughout the treatment.

To conclude, these results show that c-Abl kinase plays a pivotal role in NET generation in neutrophils and in septic lung injury. Our findings indicate that targeting c-Abl kinase activity decreases pulmonary formation of CXC chemokines in sepsis. In addition, c-Abl kinase inhibition reduced neutrophil recruitment and tissue 
injury in lung. Finally, blocking c-Abl kinase activity decreased systemic inflammation and pulmonary neutrophilia in mice with sepsis. Thus, this study not only delineates a novel signaling mechanism regulating NET formation in sepsis but also suggests that blocking c-Abl kinase might be a useful strategy to ameliorate local and systemic inflammation in sepsis.

\section{REFERENCES}

1. Levi, M. \& van der Poll, T. Inflammation and coagulation. Crit. Care Med. $\mathbf{3 8}$ S26-S34 (2010).

2. Asaduzzaman, M., Zhang, S., Lavasani, S., Wang, Y. \& Thorlacius, H. LFA-1 and MAC-1 mediate pulmonary recruitment of neutrophils and tissue damage in abdominal sepsis. Shock 30, 254-259 (2008).

3. Nagase, T. et al. Acute lung injury by sepsis and acid aspiration: a key role for cytosolic phospholipase A2. Nat. Immunol. 1, 42-46 (2000).

4. Parrillo, J. E. Pathogenetic mechanisms of septic shock. N. Engl. J. Med. 328, 1471-1477 (1993).

5. Kamochi, M. et al. P-selectin and ICAM-1 mediate endotoxin-induced neutrophil recruitment and injury to the lung and liver. Am. J. Physiol. 277, L310-L319 (1999).

6. Segal, A. W. How neutrophils kill microbes. Annu. Rev. Immunol. 23, 197-223 (2005).

7. Brinkmann, V. et al. Neutrophil extracellular traps kill bacteria. Science $\mathbf{3 0 3}$, 1532-1535 (2004).

8. Fuchs, T. A. et al. Novel cell death program leads to neutrophil extracellular traps. J. Cell Biol. 176, 231-241 (2007).

9. Saffarzadeh, M. et al. Neutrophil extracellular traps directly induce epithelial and endothelial cell death: a predominant role of histones. PLoS One 7, e32366 (2012).

10. Luo, L. et al. Proinflammatory role of neutrophil extracellular traps in abdominal sepsis. Am. J. Physiol. Lung Cell Mol. Physiol. 307, L586-L596 (2014).

11. Merza, M. et al. Neutrophil extracellular traps induce trypsin activation, inflammation, and tissue damage in mice with severe acute pancreatitis. Gastroenterology 149, 1920-1931 e1928 (2015).

12. McDonald, B., Urrutia, R., Yipp, B. G., Jenne, C. N. \& Kubes, P. Intravascular neutrophil extracellular traps capture bacteria from the bloodstream during sepsis. Cell Host Microbe 12, 324-333 (2012).

13. Jimenez-Alcazar, M. et al. Host DNases prevent vascular occlusion by neutrophil extracellular traps. Science 358, 1202-1206 (2017).

14. Hasan, Z. et al. Geranylgeranyl transferase regulates CXC chemokine formation in alveolar macrophages and neutrophil recruitment in septic lung injury. Am. J. Physiol. Lung Cell Mol. Physiol. 304, L221-L229 (2013).

15. Hasan, Z. et al. Rho-kinase signaling regulates pulmonary infiltration of neutrophils in abdominal sepsis via attenuation of CXC chemokine formation and Mac-1 expression on neutrophils. Shock 37, 282-288 (2012).

16. Wang, J. \& Pendergast, A. M. The emerging role of ABL kinases in solid tumors. Trends Cancer 1, 110-123 (2015).

17. Stephens, R. S., Johnston, L., Servinsky, L., Kim, B. S., Damarla, M. The tyrosine kinase inhibitor imatinib prevents lung injury and death after intravenous LPS in mice. Physiol. Rep. 3 (2015).

18. Yipp, B. G. et al. The lung is a host defense niche for immediate neutrophilmediated vascular protection. Sci. Immunol. 2 (2017).

19. Aman, J. et al. Effective treatment of edema and endothelial barrier dysfunction with imatinib. Circulation 126, 2728-2738 (2012).

20. Rizzo, A. N., Aman, J., van Nieuw Amerongen, G. P. \& Dudek, S. M. Targeting Abl kinases to regulate vascular leak during sepsis and acute respiratory distress syndrome. Arterioscler. Thromb. Vasc. Biol. 35, 1071-1079 (2015).

21. Nishi, $H$. et al. Neutrophil FcgammaRIIA promotes IgG-mediated glomerular neutrophil capture via Abl/Src kinases. J. Clin. Investig. 127, 3810-3826 (2017).

22. Cui, L. et al. C-Abl kinase is required for beta 2 integrin-mediated neutrophil adhesion. J. Immunol. 182, 3233-3242 (2009).

23. Tong, $\mathrm{H}$. et al. c-Abl tyrosine kinase plays a critical role in beta2 integrindependent neutrophil migration by regulating Vav1 activity. J. Leukoc. Biol. 93, 611-622 (2013)

24. Jackson, R. C. \& Radivoyevitch, T. Modelling c-Abl signalling in activated neutrophils: the anti-inflammatory effect of seliciclib. Biodiscovery 7, 4 (2013).

25. Kilkenny, C., Browne, W., Cuthill, I. C., Emerson, M. \& Altman, D. G., Group NCRRGW. Animal research: reporting in vivo experiments: the ARRIVE guidelines. Br. J. Pharmacol. 160, 1577-1579 (2010).
26. Rittirsch, D., Huber-Lang, M. S., Flierl, M. A. \& Ward, P. A. Immunodesign of experimental sepsis by cecal ligation and puncture. Nat. Protoc. 4, 31-36 (2009).

27. Borzone, G. et al. Rat and hamster species differences in susceptibility to elastaseinduced pulmonary emphysema relate to differences in elastase inhibitory capacity. Am. J. Physiol. Regul. Integr. Comp. Physiol. 293, R1342-R1349 (2007).

28. Carraway, M. S. et al. Blockade of tissue factor: treatment for organ injury in established sepsis. Am. J. Respir. Crit. Care Med. 167, 1200-1209 (2003).

29. Stoiber, W., Obermayer, A., Steinbacher, P. \& Krautgartner, W. D. The role of reactive oxygen species (ROS) in the formation of extracellular traps (ETs) in humans. Biomolecules 5, 702-723 (2015).

30. Woodring, P. J., Hunter, T. \& Wang, J. Y. Regulation of F-actin-dependent processes by the Abl family of tyrosine kinases. J. Cell Sci. 116, 2613-2626 (2003).

31. Woodring, P. J. et al. Modulation of the F-actin cytoskeleton by c-Abl tyrosine kinase in cell spreading and neurite extension. J. Cell Biol. 156, 879-892 (2002).

32. Lin, K. et al. c-Abl expression in chronic lymphocytic leukemia cells: clinical and therapeutic implications. Cancer Res. 66, 7801-7809 (2006).

33. Sridevi, P., Nhiayi, M. K. \& Wang, J. Y. Genetic disruption of Abl nuclear import reduces renal apoptosis in a mouse model of cisplatin-induced nephrotoxicity. Cell Death Differ. 20, 953-962 (2013).

34. Basit, A. et al. ICAM-1 and LFA-1 play critical roles in LPS-induced neutrophil recruitment into the alveolar space. Am. J. Physiol. Lung Cell Mol. Physiol. 291, L200-L207 (2006).

35. Remick, D. G. Pathophysiology of sepsis. Am. J. Pathol. 170, 1435-1444 (2007).

36. Jones, S. B., Lu, H. Y. \& Lu, Q. Abl tyrosine kinase promotes dendrogenesis by inducing actin cytoskeletal rearrangements in cooperation with Rho family small GTPases in hippocampal neurons. J. Neurosci. 24, 8510-8521 (2004).

37. Zandy, N. L., Playford, M. \& Pendergast, A. M. Abl tyrosine kinases regulate cellcell adhesion through Rho GTPases. Proc. Natl Acad. Sci. USA. 104, 17686-17691 (2007).

38. Hwaiz, R. et al. Rac1 signaling regulates sepsis-induced pathologic inflammation in the lung via attenuation of Mac-1 expression and CXC chemokine formation. J. Surg. Res. 183, 798-807 (2013)

39. Chislock, E. M. \& Pendergast, A. M. Abl family kinases regulate endothelial barrier function in vitro and in mice. PLoS One 8, e85231 (2013).

40. Letsiou, E. et al. Differential and opposing effects of imatinib on LPS- and ventilator-induced lung injury. Am. J. Physiol. Lung Cell Mol. Physiol. 308, L259-L269 (2015).

41. Asaduzzaman, M. et al. Platelets support pulmonary recruitment of neutrophils in abdominal sepsis. Crit. Care Med. 37, 1389-1396 (2009).

42. Bacon, K. B. \& Oppenheim, J. J. Chemokines in disease models and pathogenesis. Cytokine Growth Factor Rev. 9, 167-173 (1998).

43. $\mathrm{Li}, \mathrm{X}$. et al. Critical role of CXC chemokines in endotoxemic liver injury in mice. J. Leukoc. Biol. 75, 443-452 (2004).

44. Wan, M. X., Wang, Y., Liu, Q., Schramm, R. \& Thorlacius, H. CC chemokines induce P-selectin-dependent neutrophil rolling and recruitment in vivo: intermediary role of mast cells. Br. J. Pharmacol. 138, 698-706 (2003).

45. Zhang, X. W., Liu, Q., Wang, Y. \& Thorlacius, H. CXC chemokines, MIP-2 and KC, induce P-selectin-dependent neutrophil rolling and extravascular migration in vivo. Br. J. Pharmacol. 133, 413-421 (2001).

46. Urban, C. F., Reichard, U., Brinkmann, V. \& Zychlinsky, A. Neutrophil extracellular traps capture and kill Candida albicans yeast and hyphal forms. Cell Microbiol. 8, 668-676 (2006).

47. Kaplan, M. J. \& Radic, M. Neutrophil extracellular traps: double-edged swords of innate immunity. J. Immunol. 189, 2689-2695 (2012).

48. Wang, $Y$. et al. Neutrophil extracellular trap-microparticle complexes enhance thrombin generation via the intrinsic pathway of coagulation in mice. Sci. Rep. 8, 4020 (2018).

49. Beiter, K. et al. An endonuclease allows Streptococcus pneumoniae to escape from neutrophil extracellular traps. Curr. Biol. 16, 401-407 (2006).

50. Keshari, R. S. et al. Cytokines induced neutrophil extracellular traps formation: implication for the inflammatory disease condition. PLoS One 7, e48111 (2012)

\section{ACKNOWLEDGEMENTS}

Swedish Research Council (2017-01621) and Einar and Inga Nilsson foundation. Avin Hawez is supported by a doctoral fellowship from Regional Government of Kurdistan, Iraq. 


\section{COMPETING INTERESTS}

The authors declare no competing interests.

\section{ADDITIONAL INFORMATION}

Supplementary information The online version contains supplementary material available at https://doi.org/10.1038/s41374-021-00683-6.

Correspondence and requests for materials should be addressed to Henrik Thorlacius.

Reprints and permission information is available at http://www.nature.com/reprints

Publisher's note Springer Nature remains neutral with regard to jurisdictional claims in published maps and institutional affiliations.
Open Access This article is licensed under a Creative Commons Attribution 4.0 International License, which permits use, sharing, adaptation, distribution and reproduction in any medium or format, as long as you give appropriate credit to the original author(s) and the source, provide a link to the Creative Commons license, and indicate if changes were made. The images or other third party material in this article are included in the article's Creative Commons license, unless indicated otherwise in a credit line to the material. If material is not included in the article's Creative Commons license and your intended use is not permitted by statutory regulation or exceeds the permitted use, you will need to obtain permission directly from the copyright holder. To view a copy of this license, visit http://creativecommons. org/licenses/by/4.0/.

(c) The Author(s), under exclusive licence to United States and Canadian Academy of Pathology 2021 